\title{
Meal and Sleep Timing before and during the COVID-19 Pandemic: A Cross-Sectional Anonymous Survey Study from Sweden
}

\author{
Christian Benedict ${ }^{1, *(D)}$, Luiz Eduardo Mateus Brandão ${ }^{2}\left(\mathbb{D}\right.$, Ilona Merikanto ${ }^{3,4,5} \mathbb{D}$, Markku Partinen $^{6,7}$, \\ Bjørn Bjorvatn ${ }^{8,9}$ and Jonathan Cedernaes ${ }^{2,10, * \mathbb{D}}$
}

1 Department of Neuroscience, Sleep Science (BMC), Uppsala University, 75236 Uppsala, Sweden

2 Department of Medical Sciences, Uppsala University, 75236 Uppsala, Sweden; luiz-eduardo.mateus-brandao@medsci.uu.se

3 Department of Public Health Solutions, Finnish Institute for Health and Welfare, 00271 Helsinki, Finland; ilona.merikanto@helsinki.fi

4 Department of Psychology and Logopedics, Faculty of Medicine, University of Helsinki, 00100 Helsinki, Finland

5 Orton Orthopaedics Hospital, 00280 Helsinki, Finland

6 Helsinki Sleep Clinic, Vitalmed Research Center, 00420 Helsinki, Finland; markpart@icloud.com

7 Department of Neurosciences, Clinicum, University of Helsinki, 00100 Helsinki, Finland

8 Department of Global Public Health and Primary Care, University of Bergen, 5009 Bergen, Norway; Bjorn.Bjorvatn@uib.no

9 Norwegian Competence Center for Sleep Disorders, Haukeland University Hospital, 5021 Bergen, Norway

10 Department of Medicine, Division of Endocrinology, Metabolism, and Molecular Medicine, Feinberg School of Medicine, Northwestern University, Chicago, IL 60611, USA

check for updates

Citation: Benedict, C.; Brandão, L.E.M.; Merikanto, I.; Partinen, M.; Bjorvatn, B.; Cedernaes, J. Meal and Sleep Timing before and during the COVID-19 Pandemic: A Cross-

Sectional Anonymous Survey Study from Sweden. ClocksESleep 2021, 3, 251-258. https://doi.org/10.3390/ clockssleep3020015

Received: 1 March 2021

Accepted: 20 April 2021

Published: 22 April 2021

Publisher's Note: MDPI stays neutral with regard to jurisdictional claims in published maps and institutional affiliations.

Copyright: () 2021 by the authors. Licensee MDPI, Basel, Switzerland. This article is an open access article distributed under the terms and conditions of the Creative Commons Attribution (CC BY) license (https:// creativecommons.org/licenses/by/ $4.0 /)$.
* Correspondence: christian.benedict@neuro.uu.se (C.B.); jonathan.cedernaes@medsci.uu.se (J.C.)

\begin{abstract}
The COVID-19 pandemic and related restrictions, such as stay-at-home-orders, have significantly altered daily routines and lifestyles. Given their importance for metabolic health, we herein compared sleep and meal timing parameters during vs. before the COVID-19 pandemic based on subjective recall, in an anonymous Swedish survey. Among 191 adults (mean age: 47 years; 77.5\% females), we show that social jetlag, i.e., the mismatch in sleep midpoint between work and free days, was reduced by about 17 min during the pandemic compared with the pre-pandemic state $(p<0.001)$. Concomitantly, respondents' sleep midpoint was shifted toward morning hours during workdays $(p<0.001)$. A later daily eating midpoint accompanied the shift in sleep timing $(p=0.001)$. This effect was mainly driven by a later scheduled first meal $(p<0.001)$. No difference in the timing of the day's last meal was found $(p=0.814)$. Although our survey was limited in terms of sample size and by being cross-sectional, our results suggest that the delay in sleep timing due to the COVID-19 pandemic was accompanied by a corresponding shift in the timing of early but not late meals.
\end{abstract}

Keywords: COVID-19 pandemic; anonymous survey; meal timing; sleep timing; Sweden

\section{Introduction}

The novel coronavirus SARS-CoV-2, the causative agent of Coronavirus disease 2019 (COVID-19), was first identified at the end of 2019 in China and has since spread across the world [1]. Due to its lethality, which log-linearly rises with age [2], many countries worldwide have taken action to slow the spread of the virus, including stay-at-home, work-from-home, and social distancing orders. These social restrictions have come at a cost, including reports of reduced well-being, increasing mental health issues, and lower physical activity levels [3,4]. On the other hand, working from home may also promote certain aspects of health. Due to greater time flexibility, people may be less stressed by inflexible work schedules, thereby having the opportunity to align better their daily activities with their individual sleep/wake preference. Supporting this assumption, a study 
from Europe found that COVID-19-related restrictions, especially a lockdown, resulted in delayed bedtime, later rise times, increased sleep duration, and reduced social jetlag (shifts in sleep timing between workdays and free days) [5] - as also confirmed in a U.S. cohort [6]. Despite this evidence, it must be borne in mind that not all studies support observations of improved sleep during the early part of the pandemic [7-9]. For instance, an increasing number of psychiatric patients have been observed to complain about severe insomnia due to the pandemic and related restrictions [10]. Furthermore, according to a Greek study, sleep disturbances have been more intense for urban than rural residents [11]. Finally, in light of emerging evidence suggesting that COVID-19 can affect the brain [12], it is also possible that some changes in sleep that have been observed among people during the pandemic, may be causally resulted to effects of symptomatic and asymptomatic COVID-19.

Besides sleep, dietary choices may have also changed as a result of the COVID-19 pandemic and related restrictions. Supporting this assumption, a study from the United Arab Emirates involving 1012 subjects found more unhealthy dietary patterns during the pandemic. For instance, $46.1 \%$ of the participants consumed sweets and desserts at least once per day, and $37.1 \%$ reported consuming salty snacks (chips, crackers, and nuts) every day) [13]. In addition to diet quality, the timing of food intake may also be relevant to human health. Alterations in feeding time can uncouple the body clocks, leading to circadian misalignment, disruption in homeostasis, and disturbances in many metabolic functions [14-16]. Whether the shift of nighttime sleep to later hours during the pandemic has also shifted people's eating time window toward later hours of the day is unknown. Later eating has been associated with various health outcomes, such as an increased risk of cancer, cardiometabolic complications, reduced efficacy of weight-loss interventions, lower insulin sensitivity, and adverse body composition [17-19], although such outcomes may partly be driven by a greater mismatch with fixed work schedules (akin to similar associations observed for those with greater social jetlag).

In the present study, we used data from 191 adults from Sweden, who at the time of the pandemic were surveyed about their sleep and meal timing habits both before and during the pandemic. Our hypothesis was that adults would exhibit a shift of habitual nighttime sleep to later hours on working days (due to, e.g., flexible work schedules) and, consequently, a reduction in social jetlag. We also hypothesized that delayed sleep schedules would occur concomitantly with a later onset and offset of the habitual meal timing windows.

\section{Results}

\subsection{Cohort Characteristics}

The anonymous survey was available online, in Swedish, between June and August 2020 (more details about the structure of the survey can be found in ref. [20]). It was administered through the Qualtrics platform (https://www.qualtrics.com accessed on 25 June 2020) and publicly accessible (until 10 August 2020). Of the questionnaire's 869 respondents, 191 provided the full information required for the present analysis (for more details on exclusion criteria, see Table S1). Participation in the online survey was voluntarily, could be stopped at any time point by the respondent, and was not reimbursed. The final sample was on average 47.2 (13.1) years old, slightly overweight with BMI around $25.2(4.7)(27.2 \%$ of respondents were overweight and $16.8 \%$ were obese), mainly female $(77.5 \%)$, and the majority was either married or cohabiting (70.7\%). A $13.1 \%$ share of the respondents stated having a definitely morning circadian preference, and $27.2 \%$ indicated a more morning than evening circadian preference. Additionally, $18.8 \%$ reported an intermediate chronotype. Finally, $24.1 \%$ stated that they had a more evening than morning circadian preference, and $16.8 \%$ stated having a definitely evening circadian preference. 


\subsection{Sleep before and Amid the Pandemic}

Both before and during the pandemic, on working days, participants went earlier to bed, woke up earlier, exhibited an earlier sleep midpoint, and slept shorter compared with free days $(p<0.001$ for all Wilcoxon signed-rank test comparisons; Table 1). Similarly, compared with free days, participants reported earlier time points for the first and last meal of the day (resulting in an earlier eating midpoint) and longer eating time windows on working days ( $p<0.001$ for all Wilcoxon signed-rank test comparisons). Again, these differences were observed both before and during the pandemic (Table 1).

Table 1. Sleep and meal timing before and amid the COVID-19 pandemic, on work and free days. Social jetlag was calculated by subtracting the sleep midpoint of workdays from the free days' sleep midpoint. Eating jetlag was calculated by subtracting the meal midpoint of workdays from the free days' meal midpoint. Due to skewness of the data, comparisons between the time points were analyzed with the non-parametric Wilcoxon signed-rank test. A $p$-value smaller than 0.0028 was considered significant (shown in bold; $p=0.05$ divided by 18 comparisons). Data are shown as mean \pm standard deviation (SD). Abbreviations: hh:mm, military time; hr.min, hours + minutes.

\begin{tabular}{ccccc}
\hline Parameter & Pre-Pandemic & Pandemic & Z Value & $p$ Value \\
\hline Workdays & Mean (SD) & Mean (SD) & & \\
Bedtime (hh:mm) & $22: 40(00: 55)$ & $22: 53(01: 05)$ & -3.910 & $<0.001$ \\
Waking time (hh:mm) & $06: 17(00: 56)$ & $06: 27(01: 08)$ & -2.532 & 0.011 \\
Sleep midpoint (hh:mm) & $02: 29(00: 49)$ & $02: 40(00: 57)$ & -4.204 & $<0.001$ \\
Time in bed (hr.min) & $7.38(0.56)$ & $7.34(1.08)$ & -1.035 & 0.301 \\
First meal (hh:mm) & $07: 25(01: 16)$ & $07: 48(01: 22)$ & -6.013 & $<0.001$ \\
Last meal (hh:mm) & $18: 20(00: 59)$ & $18: 21(01: 02)$ & -0.235 & 0.814 \\
Eating midpoint (hh:mm) & $12: 52(00: 50)$ & $13: 04(00: 55)$ & -4.715 & $<0.001$ \\
Eating time window (hr.min) & $10.55(1: 32)$ & $10.34(1: 35)$ & -4.939 & $<0.001$ \\
\hline Free days & & & & \\
Bedtime (hh:mm) & $23: 17(01: 03)$ & $23: 18(01: 13)$ & -0.033 & 0.973 \\
Waking time (hh:mm) & $07: 43(1.27)$ & $07: 34(01: 23)$ & -2.008 & 0.045 \\
Sleep midpoint (hh:mm) & $03: 30(01: 02)$ & $03: 26(01: 09)$ & -1.456 & 0.145 \\
Time in bed (hr.min) & $8.26(1.05)$ & $8.16(1.14)$ & -1.987 & 0.047 \\
First meal (hh:mm) & $08: 50(01: 22)$ & $08: 59(01: 17)$ & -3.239 & 0.001 \\
Last meal (hh:mm) & $18: 38(01: 02)$ & $18: 42(01: 02)$ & -1.586 & 0.113 \\
Eating midpoint (hh:mm) & $13: 44(00: 59)$ & $13.50(00: 57)$ & -3.158 & 0.002 \\
Eating time window (hr.min) & $9.47(1.23)$ & $9.43(1.23)$ & -1.719 & 0.086 \\
Social jetlag (hr.min) & $1.04(0.49)$ & $0.46(0.47)$ & -6.020 & $<0.001$ \\
Eating jetlag (hr.min) & $0.52(0.53)$ & $0.47(0.49)$ & -0.111 & 0.912 \\
\hline
\end{tabular}

As summarized in Table 1 and Figure 1, on workdays but not free days, respondents went to bed significantly later during the pandemic compared to the pre-pandemic period ( 13 min on average). Although the waking time was delayed by approximately $10 \mathrm{~min}$ on working days during the pandemic, following adjustment for multiple comparisons, the comparison with pre-pandemic waking time did not reach significance. We nonetheless found that the sleep midpoint on workdays was on average approximately $11 \mathrm{~min}$ later. No difference in sleep midpoint between pre-pandemic and pandemic on free days was found. Indicating that sleep times between work and free days were better aligned when compared with the pre-pandemic period, social jetlag significantly decreased during the pandemic ( -17 min on average), but no difference in time in bed was observed. 

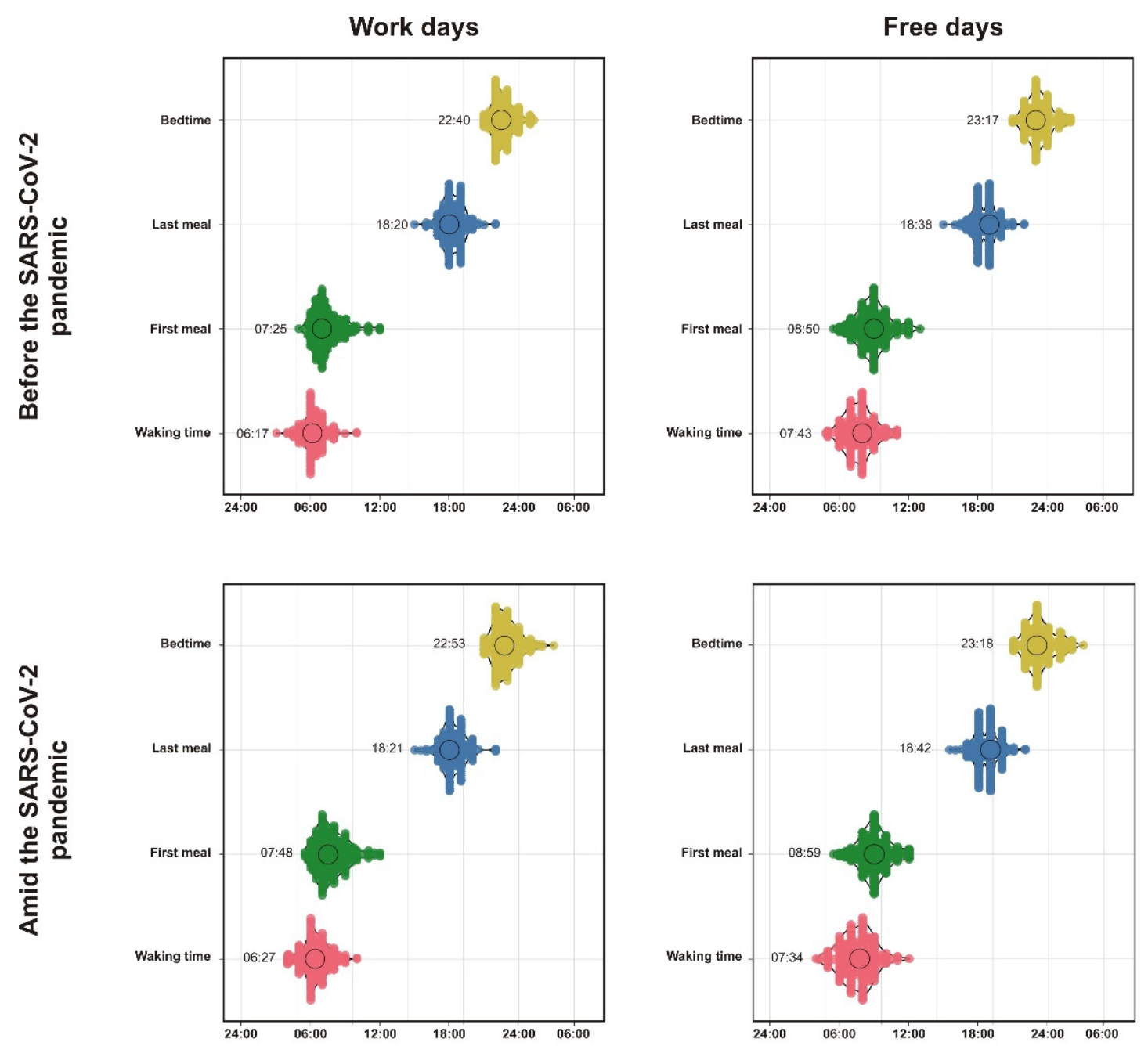

Figure 1. Meal and sleep timing variables before and amid the COVID-19 pandemic, split by work and free days. Mean value (in military time) is shown next to each figure element; y-axis shows frequency over clock time.

\subsection{Meal Timing before and Amid the Pandemic}

Respondents scheduled their first meal about $23 \mathrm{~min}$ later on workdays and $9 \mathrm{~min}$ later on free days during the pandemic compared to the pre-pandemic period (Table 1 and Figure 1). In contrast, the timing of the last meal did not differ significantly between the pandemic vs. pre-pandemic period. Mainly driven by the delayed onset of the first meal, the eating midpoint was about 12 min later on workdays and 6 min later on free days during the pandemic compared with before the pandemic. We also observed that the length of the eating time window was significantly shorter on workdays during the pandemic ( $-21 \mathrm{~min})$. Finally, eating jet lag did not differ between the pandemic and pre-pandemic periods.

Before and during the pandemic, significant correlations were observed between the sleep and eating midpoints, both on working and free days (Table 2), supporting the notion that meal timing is associated with sleep timing. 
Table 2. Spearman correlation between sleep and eating midpoints before and during the pandemic. The table reports Spearman rank-order correlation coefficients. ${ }^{* *} p$ value smaller than 0.0125 (Bonferroni corrected). Abbreviations: N/A, not applicable.

\begin{tabular}{|c|c|c|c|c|c|c|}
\hline & & & \multicolumn{2}{|c|}{ Pre-Pandemic } & \multicolumn{2}{|c|}{ Pandemic } \\
\hline & & & \multicolumn{2}{|c|}{ Eating Midpoint } & \multicolumn{2}{|c|}{ Eating Midpoint } \\
\hline & & & Workdays & Free Days & Workdays & Free Days \\
\hline \multirow{2}{*}{ Pre-pandemic } & \multirow{2}{*}{ Sleep midpoint } & Workdays & 0.540 ** & $\mathrm{N} / \mathrm{A}$ & $\mathrm{N} / \mathrm{A}$ & $\mathrm{N} / \mathrm{A}$ \\
\hline & & Free days & $\mathrm{N} / \mathrm{A}$ & $0.697^{* *}$ & $\mathrm{~N} / \mathrm{A}$ & $\mathrm{N} / \mathrm{A}$ \\
\hline \multirow{2}{*}{ Pandemic } & \multirow{2}{*}{ Sleep midpoint } & Workdays & $\mathrm{N} / \mathrm{A}$ & $\mathrm{N} / \mathrm{A}$ & $0.478 * *$ & $\mathrm{~N} / \mathrm{A}$ \\
\hline & & Free days & $\mathrm{N} / \mathrm{A}$ & $\mathrm{N} / \mathrm{A}$ & $\mathrm{N} / \mathrm{A}$ & $0.618^{* *}$ \\
\hline
\end{tabular}

\section{Discussion}

In the present study, we found a relatively small but significant effect of the COVID-19 pandemic on sleep schedules. Compared to before, people went about 13 min later to bed during the pandemic. Reflecting how meal timing may be inherently tied to the sleep/wake cycle, we observed that a later bedtime and sleep midpoint during the COVID-19 pandemic were accompanied by a later scheduled first meal on workdays and free days. Notably, in contrast, the timing of the last meal amid the pandemic did not differ compared to before the pandemic. As respondents' main change in eating patterns was a later start to their first meal of the day (meal onset), their eating time window was shorter on working days during the pandemic, and the evening fasting period was prolonged on working days. A shorter daily eating time window has been linked to improved metabolic health among obese and overweight subjects [21]. Longer duration of pre-sleep fasting may reduce the risk of gastroesophageal reflux disease [22]. Finally, delaying the first meal of the day prolongs the overnight fast (i.e., the time between the last meal of the preceding day and the first meal of the current day), which has been associated with a reduced likelihood of being overweight or obese [23]. Some of these effects may occur through modulation of the diurnal cortisol profile and meal-induced thermogenesis [24,25].

There were limitations to this study. There could have been bias in the range of responses, as our survey was distributed online without the ability to ensure responses covering a broad range of demographics. Our data were, furthermore, based on participant recall, which is also subject to bias. Many subjects who completed the survey were excluded for missing data, which may be explained by the lack of financial reimbursement for survey participation. Additionally, we excluded respondents with irregular working hours (e.g., night shift workers). This loss of data might have introduced selection bias. Residual confounding due to factors such as seasonality should also be taken into consideration when interpreting our findings.

Another important limitation was that we did not examine whether dietary choices differed between, before, and during the pandemic. A recent survey study from Italy revealed that respondents exhibited less healthy dietary choices during the pandemic than before, including but not limited to increased intake of simple sugar and sweets and decreased intake of legumes [26]. Thus, it is unclear whether possible health benefits related to a shorter eating time window and longer overnight fast, as observed in our study, might be offset by poor dietary choices during the pandemic.

Although we found that bedtime and sleep midpoint were delayed, sleep duration remained unchanged, which contrasts with previous reports of increased sleep duration during the COVID-19 pandemic [5,6]. In this context, it is important to bear in mind that in Sweden, sports events and large gatherings were banned, but business, cafes, and shops remained open for people during the period of our data collection in 2020 . Looser restrictions and a significant number of daily COVID-19-related deaths may have contributed to poorer mental health and wellbeing in Sweden. As suggested by a Swedish survey study, $45.6 \%$ reported symptoms reflecting significant problems in one or more 
areas of their mental health, including depression, anxiety, or insomnia [27]. These adverse effects on mental health may have weakened possible positive effects of work schedule flexibility due to work at home recommendations on sleep duration.

\section{Materials and Methods}

\subsection{Survey Procedure}

With the primary aim to investigate the impact of the SARS-CoV-2 pandemic on sleep and circadian rhythms, an anonymous online survey study was performed across 14 countries/areas during 2020 (International COVID-19 Sleep Study, ref. [20]). Respondents were asked about the exact going to bed and waking up times on both work and free days before and during the pandemic. Cronbach's alpha indicated acceptable internal consistency for questions on bedtime and waking time on working and free days (alpha $=0.897$ ). Thus, we could calculate the time in bed and social jetlag (the difference in sleep midpoint between work and free days, ref. [28]). In Sweden only, respondents were also asked about meal timing. Specifically, the participants indicated the exact clock time of the first and last main meal on work and free days separately, both before and during the pandemic (Crohnbach's alpha $=0.818$ ). We then estimated the following (pre)pandemic meal timing characteristics: time of the first and last meal, the midpoint of the eating time window, eating jet lag (the difference in eating midpoint between work and free days), and length of eating time window. Information about the existence of the anonymous survey was circulated on TV, Internet, and social media platforms. The survey was presented as questionnaire aimed at investigating sleep and associated lifestyle habits during the COVID-19 pandemic. No information was provided about this paper's expected outcome. Between 25 June 2020, and 10 August 2020, participants were able to respond to the questionnaire, which was presented in Swedish, via the online platform Qualtrics. Following exclusions (specified in Table S1), complete survey data of 191 anonymous respondents were available for analysis.

\subsection{Statistical Analysis}

Statistical analyses were performed using SPSS version 24 (SPSS Inc., Chicago, IL, USA). Due to the skewness of the data (confirmed by significant Shapiro-Wilk tests), we used the non-parametric Wilcoxon signed-rank test to examine whether sleep and meal timing variables differed within subjects between the pre-pandemic and pandemic periods, as well as between work and free days. Given the moderate sample size of the present study, our main analysis was restricted to the pandemic's effects on meal and sleep timing parameters in the full cohort. Exploratory analyses, stratified by age, sex, and BMI—all known to affect circadian rhythms [29]—can be found in Tables S2-S4. Overall, a $p$ value $<0.05$ was considered significant. Where appropriate, Bonferroni correction was applied when testing multiple pairwise comparisons. If not otherwise stated, data are shown as mean (SD).

\section{Conclusions}

Our study suggests that later sleep times, as seen during the COVID-19 pandemic, prolong daily meal onset latency. The observation that the timing of the last meal remained unaffected by later sleep times could suggest that other variables not measured herein, such as the habit of having a regular dinner with the spouse or family, may play a more prominent role in determining when the last meal is consumed. Our findings must be confirmed by data collected in other countries and in larger populations that allow for more detailed analyses of possible confounding factors.

Supplementary Materials: The following are available online at https:/ / www.mdpi.com/article / 10.3390/clockssleep3020015/s1, Table S1: Exclusions; Table S2: Sleep and meal timing before and amid the COVID-19 pandemic, split by age; Table S3: Sleep and meal timing before and amid the COVID-19 pandemic, split by gender; and Table S4: Sleep and meal timing before and amid the COVID-19 pandemic, split by BMI. 
Author Contributions: Conceptualization, M.P., B.B., I.M., and J.C.; methodology, J.C.; formal analysis, C.B.; data curation, C.B. and J.C.; writing-original draft preparation, C.B.; writing-review and editing, L.E.M.B., I.M., M.P., B.B., and J.C.; visualization, C.B.; supervision, J.C. All authors have read and agreed to the published version of the manuscript.

Funding: This research received no specific external funding. The authors' work is funded by the Novo Nordisk Foundation (NNF19OC0056777), Swedish Brain Research Foundation (FO2020-0044 \& FO2020-0334), the Swedish Foundation for Medical Research, the Göran Gustafsson Foundation, and the Swedish Cancer Foundation.

Institutional Review Board Statement: The anonymous online survey made re-identification of the respondents impossible. Thus, no ethical or legal clearance was required according to Swedish law.

Informed Consent Statement: Participants' consent was waived due to anonymity of the survey. Due to the setup of our online survey, re-identification of the respondents was impossible. Before filling out the survey, participants were informed that the collected anonymous survey data would be used for research purposes only.

Data Availability Statement: Upon request from C.B. or J.C., the data can be shared with researchers.

Acknowledgments: We would like to thank Agneta Wetterberg, Per Cedernaes, and Laura Kortesoja for their help with the questionnaire.

Conflicts of Interest: None of the authors except for C.B. has conflicts of interest to report. C.B. reports that he served as a paid member of a scientific advisory board for Repha GmBH, Langenhagen, Germany. C.B. declares no other conflict of interest. The funders had no role in the design of the study; in the collection, analyses, or interpretation of data; in the writing of the manuscript, or in the decision to publish the results.

\section{References}

1. Wu, A.; Peng, Y.; Huang, B.; Ding, X.; Wang, X.; Niu, P.; Meng, J.; Zhu, Z.; Zhang, Z.; Wang, J.; et al. Genome Composition and Divergence of the Novel Corona-virus (2019-nCoV) Originating in China. Cell Host Microbe 2020, 27, 325-328. [CrossRef]

2. O’Driscoll, M.; Dos Santos, G.R.; Wang, L.; Cummings, D.A.T.; Azman, A.S.; Paireau, J.; Fontanet, A.; Cauchemez, S.; Salje, H. Age-specific mortality and immunity patterns of SARS-CoV. Nat. Cell Biol. 2021, 590, 140-145. [CrossRef]

3. Le, H.T.; Lai, A.J.X.; Sun, J.; Hoang, M.T.; Vu, L.G.; Pham, H.Q.; Nguyen, T.H.; Tran, B.X.; Latkin, C.A.; Le, X.T.T.; et al. Anxiety and Depression Among People Under the Nationwide Partial Lockdown in Vietnam. Front. Public Health 2020, 8, 589359. [CrossRef] [PubMed]

4. Violant-Holz, V.; Gallego-Jiménez, M.G.; González-González, C.S.; Muñoz-Violant, S.; Rodríguez, M.J.; Sansano-Nadal, O.; Guerra-Balic, M. Psychological Health and Physical Activity Levels during the COVID-19 Pandemic: A Systematic Review. Int. J. Environ. Res. Public Health 2020, 17, 9419. [CrossRef]

5. Blume, C.; Schmidt, M.H.; Cajochen, C. Effects of the COVID-19 lockdown on human sleep and rest-activity rhythms. Curr. Biol. 2020, 30, R795-R797. [CrossRef] [PubMed]

6. Wright, K.P., Jr.; Linton, S.K.; Withrow, D.; Casiraghi, L.; Lanza, S.M.; Iglesia, H.; Vetter, C.; Depner, C.M. Sleep in uni-versity students prior to and during COVID-19 Stay-at-Home orders. Curr. Biol. 2020, 30, R797-R798. [CrossRef] [PubMed]

7. Partinen, M. Sleep research in 2020: COVID-19-related sleep disorders. Lancet Neurol. 2021, 20, 15-17. [CrossRef]

8. Jahrami, H.; Bahammam, A.S.; Bragazzi, N.L.; Saif, Z.; Faris, M.; Vitiello, M.V. Sleep problems during the COVID-19 pandemic by population: A systematic review and meta-analysis. J. Clin. Sleep Med. 2021, 17, 299-313. [CrossRef]

9. Li, Y.; Qin, Q.; Sun, Q.; Sanford, L.D.; Vgontzas, A.N.; Tang, X. Insomnia and psychological reactions during the COVID-19 outbreak in China. J. Clin. Sleep Med. 2020, 16, 1417-1418. [CrossRef]

10. Hao, F.; Tan, W.; Jiang, L.; Zhang, L.; Zhao, X.; Zou, Y.; Hu, Y.; Luo, X.; Jiang, X.; McIntyre, R.S.; et al. Do psychiatric patients experience more psychiatric symp-toms during COVID-19 pandemic and lockdown? A case-control study with service and research implications for im-munopsychiatry. Brain Behav. Immun. 2020, 87, 100-106. [CrossRef]

11. Anastasiou, E.; Duquenne, M.N. What about the "Social Aspect of COVID"? Exploring the Determinants of Social Isola-tion on the Greek Population during the COVID-19 Lockdown. Soc. Sci. 2021, 10, 27. [CrossRef]

12. De Berardis, D. How concerned should we be about neurotropism of SARS-Cov-2? A brief clinical consideration of the possible psychiatric implications. CNS Spectr. 2020, 10, 1-6. [CrossRef]

13. Ismail, L.C.; Osaili, T.M.; Mohamad, M.N.; Al Marzouqi, A.; Jarrar, A.H.; Abu Jamous, D.O.; Magriplis, E.; Ali, H.I.; Al Sabbah, H.; Hasan, H.; et al. Eating Habits and Lifestyle during COVID-19 Lockdown in the United Arab Emirates: A Cross-Sectional Study. Nutrients 2020, 12, 3314. [CrossRef]

14. Parsons, M.J.; Moffitt, T.; Gregory, A.M.; Goldman-Mellor, S.; Nolan, P.M.; Poulton, R.; Caspi, A. Social jetlag, obesity and metabolic disorder: Investigation in a cohort study. Int. J. Obes. 2015, 39, 842-848. [CrossRef] 
15. Cedernaes, J.; Waldeck, N.; Bass, J. Neurogenetic basis for circadian regulation of metabolism by the hypothalamus. Genes Dev. 2019, 33, 1136-1158. [CrossRef]

16. Da Silva, F.R.; Junior, A.H.L.; Brant, V.M.; Lôbo, I.L.B.; Lancha, L.O.P.; Silva, A.; De Mello, M.T. The effects of COVID-19 quarantine on eating and sleeping behaviors. Nutrients 2020, 45, 25. [CrossRef]

17. Kogevinas, M.; Espinosa, A.; Castelló, A.; Gómez-Acebo, I.; Guevara, M.; Martin, V.; Amiano, P.; Alguacil, J.; Peiro, R.; Moreno, V.; et al. Effect of mistimed eating pat-terns on breast and prostate cancer risk (MCC-Spain Study). Int. J. Cancer 2018, 143, 2380-2389. [CrossRef] [PubMed]

18. Dashti, H.S.; Gómez-Abellán, P.; Qian, J.; Esteban, A.; Morales, E.; Scheer, F.A.J.L.; Garaulet, M. Late eating is associated with cardiometabolic risk traits, obesogenic behaviors, and impaired weight loss. Am. J. Clin. Nutr. 2021, 113, 154-161. [CrossRef] [PubMed]

19. McHill, A.W.; Phillips, A.J.; Czeisler, C.; Keating, L.; Yee, K.; Barger, L.K.; Garaulet, M.; Scheer, F.A.; Klerman, E.B. Later circadian timing of food intake is associated with increased body fat. Am. J. Clin. Nutr. 2017, 106, 1213-1219. [CrossRef]

20. Partinen, M.; Bjorvatn, B.; Holzinger, B.; Chung, F.; Penzel, T.; Espie, C.A.; Morin, C.M. ICOSS-collaboration group Sleep and circadian problems during the coronavirus disease 2019 (COVID-19) pandemic: The International COVID-19 Sleep Study (ICOSS). J. Sleep Res. 2021, 30, e13206. [CrossRef] [PubMed]

21. Wilkinson, M.J.; Manoogian, E.N.; Zadourian, A.; Lo, H.; Fakhouri, S.; Shoghi, A.; Wang, X.; Fleischer, J.G.; Navlakha, S.; Panda, S.; et al. Ten-hour time-restricted eating reduces weight, blood pressure, and atherogenic lipids in patients with metabolic syndrome. Cell Metab. 2020, 31, 92-104.e5. [CrossRef]

22. Fujiwara, Y.; Machida, A.; Watanabe, Y.; Shiba, M.; Tominaga, K.; Watanabe, T.; Oshitani, N.; Higuchi, K.; Arakawa, T. Association Between Dinner-to-Bed Time and Gastro-Esophageal Reflux Disease. Am. J. Gastroenterol. 2005, 100, 2633-2636. [CrossRef] [PubMed]

23. Xiao, Q.; Bauer, C.; Layne, T.; Playdon, M. The association between overnight fasting and body mass index in older adults: The interaction between duration and timing. Int. J. Obes. 2021, 45, 555-564. [CrossRef] [PubMed]

24. Bandín, C.; Scheer, F.A.J.L.; Luque, A.J.; Ávila-Gandía, V.; Zamora, S.; Madrid, J.; Gómez-Abellán, P.; Garaulet, M. Meal timing affects glucose tolerance, substrate oxidation and circadian-related variables: A randomized, crossover trial. Int. J. Obes. 2015, 39, 828-833. [CrossRef] [PubMed]

25. Jamshed, H.; Beyl, R.A.; Della Manna, D.L.; Yang, E.S.; Ravussin, E.; Peterson, C.M. Early time-restricted feeding improves 24-hour glucose levels and affects markers of the circadian clock, aging, and autophagy in humans. Nutrients 2019, 11, 1234. [CrossRef]

26. Cicero, A.F.; Fogacci, F.; Giovannini, M.; Mezzadri, M.; Grandi, E.; Borghi, C. COVID-19-Related Quarantine Effect on Die-tary Habits in a Northern Italian Rural Population: Data from the Brisighella Heart Study. Nutrients 2021, 13, 309. [CrossRef] [PubMed]

27. McCracken, L.M.; Badinlou, F.; Buhrman, M.; Brocki, K.C. Psychological impact of COVID-19 in the Swedish population: De-pression, anxiety, and insomnia and their associations to risk and vulnerability factors. Eur. Psychiatry 2020, 63, e81. [CrossRef]

28. Wittmann, M.; Dinich, J.; Merrow, M.; Roenneberg, T. Social Jetlag: Misalignment of Biological and Social Time. Chronobiol. Int. 2006, 23, 497-509. [CrossRef]

29. Roenneberg, T.; Wirz-Justice, A.; Merrow, M. Life between Clocks: Daily Temporal Patterns of Human Chronotypes. J. Biol. Rhythm. 2003, 18, 80-90. [CrossRef] 